\title{
Green Route for Efficient Synthesis of Novel Amino Acid Schiff Bases as Potent Antibacterial and Antifungal Agents and Evaluation of Cytotoxic Effects
}

\author{
Harshita Sachdeva, ${ }^{1}$ Rekha Saroj, ${ }^{1}$ Sarita Khaturia, ${ }^{1}$ \\ Diksha Dwivedi, ${ }^{1}$ and Om Prakash Chauhan ${ }^{2}$ \\ ${ }^{1}$ Department of Chemistry, Faculty of Engineering and Technology, Mody Institute of Technology and Science, \\ Lakshmangarh, Rajasthan 332311, India \\ ${ }^{2}$ Maharshi Dayanand Saraswati University, Ajmer, Rajasthan 305009, India \\ Correspondence should be addressed to Harshita Sachdeva; drhmsachdevaster@gmail.com
}

Received 7 May 2013; Revised 2 November 2013; Accepted 20 November 2013; Published 30 January 2014

Academic Editor: Marijan Kocevar

Copyright @ 2014 Harshita Sachdeva et al. This is an open access article distributed under the Creative Commons Attribution License, which permits unrestricted use, distribution, and reproduction in any medium, provided the original work is properly cited.

Green chemical one-pot multicomponent condensation reaction of substituted $1 H$-indole-2,3-diones (1), various amino acids (2), and thiosemicarbazide (3) is found to be catalyzed by lemon juice as natural acid using water as a green solvent to give the corresponding Schiff bases (4) in good to excellent yields. This method is experimentally simple, clean, high yielding, and green, with reduced reaction times. The product is purified by simple filtration followed by washing with water and drying process. The synthesized compounds are characterized by FT-IR, ${ }^{13}$ CNMR, and ${ }^{1}$ HNMR spectroscopy and are screened for their antifungal activity against Aspergillus niger, Penicillium notatum, Fusarium oxysporum, Alternaria brassicicola, Chaetomium orium, and Lycopodium sp. and antibacterial activity against Gram-positive bacteria, Bacillus licheniformis, Staphylococcus aureus, and Micrococcus luteus, and Gram-negative bacteria, Pseudomonas aeruginosa and Escherichia coli. Compounds have also been evaluated for cytotoxic effects against human colon cancer cell line Colo205.

\section{Introduction}

In recent years, environmentally benign synthetic methods have received considerable attention and some green protocols have been developed $[1,2]$. There has been a large emphasis both in the chemical industry and in academic research on the development of environmentally benign solvents and reaction conditions. Organic synthetic procedures use organic solvents like benzene and chlorinated hydrocarbons, which have created havoc to the environment because of their toxic and volatile nature $[3,4]$.

To reduce such disasters there is a need to use a safer reaction medium like water. Water is nontoxic, safe, and cheap and never leads to menace to the environment [5-10]. The use of water as a solvent [11-13] is undoubtedly the best alternative as there are generally no harsh reaction conditions and no need of vigorous drying of the solvents.
Schiff bases have been playing vital roles in the preparation of a large number of industrial and biologically active compounds via closure, cycloaddition, and replacement reactions. They are also known for their diversified biological activities such as anti-inflammatory, allergic inhibitors, radical scavenging, analgesic and antioxidative action, and so forth [14-16]. Further, the incorporation of oxindole nuclei is an important synthetic strategy in drug discovery. Oxindoles have shown an extensive range of biological effects, including antibacterial, antifungal, anticonvulsant, antiviral, and antiproliferative activity [17]. On the other hand, heterocyclic compounds possessing 1,3,4-thiadiazole ring system show antifungal, bacteriostatic, anthelmintic [18] effect as well as depression effect on central nervous system [19]. Palaska et al. [20] reported 1,3,4-thiadiazole derivatives that exhibited analgesic and anti-inflammatory activities. 
Considering the numerous applications of Schiff bases in various fields of chemistry, there has been tremendous interest in developing efficient methods for their preparation. Synthesis of Schiff base is often carried out with acid catalyst and generally by refluxing the mixture of aldehyde (or ketone) and amine in organic medium [21]. Classical organic synthesis of Schiff bases commonly meets the problem of removing solvents from the reaction mixture [22]. To overcome the difficulties in the removal of water, alternative method has been employed in which Lewis acid is used as catalyst which accelerates nucleophilic attack of amines on carbonyl carbon as well as serving as dehydrating agent for removal of the water. Several methods have been reported in the literature in which Lewis acids were used as catalysts such as $\mathrm{ZnCl}_{2}$ [23], $\mathrm{TiCl}_{4}$ [24], alumina [25], and $\mathrm{P}_{2} \mathrm{O}_{5}$ [26] and also by using materials like hydrotalcite [27]. Environmentally benign methods for the synthesis of Schiff bases have also been reported in the literature [28-32]. Although reported methodologies have their own advantages, they are overled by prolonged reaction time, the high reaction temperatures, an excess of costly dehydrating reagents/catalysts, moisture sensitive catalysts, special apparatus, and so forth.

It has been observed that the incorporation of more than one bioactive heterocyclic moiety into a single framework may result in the production of novel heterocycles with enhanced bioactivity.Keeping in view the diverse biological activities associated with oxindole and thiadiazole, we have synthesized Schiff bases incorporating these moieties and the synthesized compounds were evaluated for their antibacterial, antifungal, and cytotoxic effects. Moreover, Schiff bases have been synthesized in aqueous medium under the framework of green chemistry in moderate to good yields and short reaction time. The condensation reaction occurs very efficiently in the presence of water and the product can be isolated simply by filtration without the need of any further purification.

\section{Results and Discussion}

In continuation of our work to develop green methodologies for the synthesis of organic compounds [33-36], herein we wish to report a mild and highly efficient procedure for the synthesis of 3-[1-(5-amino-[1,3,4]-thiadiazol-2yl)substitutedethylimino]-5-substituted-2,3-dihydro-indol2-one derivatives (4) using water as a green solvent and citric acid as a green catalyst (Scheme 1). 4 was synthesized by the one-pot multicomponent condensation reaction of substituted $1 H$-indole-2,3diones (1), various amino acids (2), and thiosemicarbazide (3) in the presence of lemon juice. The role of lemon juice in catalyzing the reaction was demonstrated by the lack of Schiff base formation when the reaction was carried out in the absence of catalyst. As lemon juice is acidic in nature $(\mathrm{pH} \approx 2-3)$ and percentage of citric acid (5-7\%) is more than other acids, it works as acid catalyst for Schiff base formation [37]. Using this methodology these reactions were completed in shorter reaction times (2-3 hrs) with yields of the product ranging from 70 to $86 \%$. For the present reaction, we have used extract of Citrus limonum species of lemon as natural catalyst. The main ingredients of lemon juice are moisture (85\%), carbohydrates $(11.2 \%)$, citric acid (5-7\%), protein $(1 \%)$, vitamin C $(0.5 \%)$, fat $(0.9 \%)$, minerals $(0.3 \%)$, fibers $(1.6 \%)$, and some other organic acids.

This green route method requires simple work-up procedure, that is, simple filtration to isolate the products as they are insoluble in water and the desired products are obtained with satisfactory yields without any further purification. Considering the reaction time with water as solvent and yield of products this process was selected as green, environmental benign, clean, and safe to promote the synthesis of various Schiff's bases (Table 1). This acceleration has been attributed to many factors, including the hydrophobic effect $[38,39]$, enhanced hydrogen bonding in the transition state [40], and the high cohesive energy density of water $[41,42]$.

Initially, we attempted to synthesize Schiff bases incorporating oxindole and 1,3,4-thiadiazole moieties in water by the reaction of 1,2 , and 3 in the presence of $\mathrm{POCl}_{3}$. Reaction gets completed in 5-6 hrs resulting in the formation of 4 with yields ranging from 60 to $70 \%$. After the successful completion of reaction in water in the presence of $\mathrm{POCl}_{3}$, we decided to synthesize Schiff bases by employing lemon juice $(2 \mathrm{~mL})$ as green catalyst for green approach (Scheme 1) resulting in quantitative yield (70-86\%) of the corresponding Schiff bases within 2 to 3 hrs. However, in the absence of lemon juice, spiro(indole-triazoles) are formed [43]. We have also carried out this reaction in the presence of citric acid but the yield of the product (4) was found to be low (60$70 \%$ ) as compared to that carried out using lemon juice as a green catalyst (70-86\%). All the synthesized compounds were found to be chiral [44]. The occurrence of $\mathrm{E} / \mathrm{Z}$ isomerism at the imine band of the molecules was suggested by Sari and Gürkan [45].

To establish the scope and limitations of lemon juice as a catalyst for Schiff base formation, structurally diverse $1 \mathrm{H}$-indole-2,3-diones were treated with variously substituted amino acids under the catalytic influence of lemon juice and the results are summarized in Table 1. The purity of the compounds was checked by TLC using silica gel-G as adsorbent. The identity of the products obtained was confirmed by their IR, ${ }^{13} \mathrm{C} \mathrm{NMR}$, and ${ }^{1} \mathrm{H}$ NMR spectral studies. The stretching bands derived from $-\mathrm{OH}$ and $\mathrm{C}=\mathrm{O}$ of carboxylic acid were disappeared with the appearance of new two bands at 3259 and $3157 \mathrm{~cm}^{-1}$ due to asymmetric and symmetric stretching vibrations of $\mathrm{NH}_{2}$ group. This evidence clearly confirmed the formation of compound 4 .

In summary, it can be stated that the present green synthetic protocol is highly efficient as it avoids the use of hazardous solvents at any stage of the reaction. As cited in Scheme 1, not only does the use of water as solvent allow for rapid reactions, but also the products are often insoluble in water, facilitating their ready isolation.

\section{Experimental Section}

3.1. General. Reagents and solvents were obtained from commercial sources and used without further purification. Melting points were determined on a Toshniwal apparatus. 
TABLE 1: Results of synthesis of $\mathbf{4 a - p}$ in the presence of lemon juice as natural acid.

\begin{tabular}{|c|c|c|c|c|c|}
\hline Entry & Product & Yield (\%) & M.P. $\left({ }^{\circ} \mathrm{C}\right)$ & Time (hrs) & Color \\
\hline $4 a$ & & 86 & 195 & $2-3$ & Brown \\
\hline $4 b$ & & 74 & 170 & $2-3$ & Brown \\
\hline $4 \mathrm{c}$ & & 78 & 103 & $2-3$ & $\begin{array}{c}\text { Dark } \\
\text { brown }\end{array}$ \\
\hline $4 d$ & & 75 & 190 & $2-3$ & $\begin{array}{c}\text { Dark } \\
\text { brown }\end{array}$ \\
\hline $4 e$ & & 74 & 170 & $2-3$ & Brown \\
\hline $4 \mathrm{f}$ & & 77 & 190 & $2-3$ & Brown \\
\hline $4 g$ & & 70 & 98 & $2-3$ & Brown \\
\hline $4 h$ & & 72 & 140 & $2-3$ & Red \\
\hline
\end{tabular}


TABLE 1: Continued.

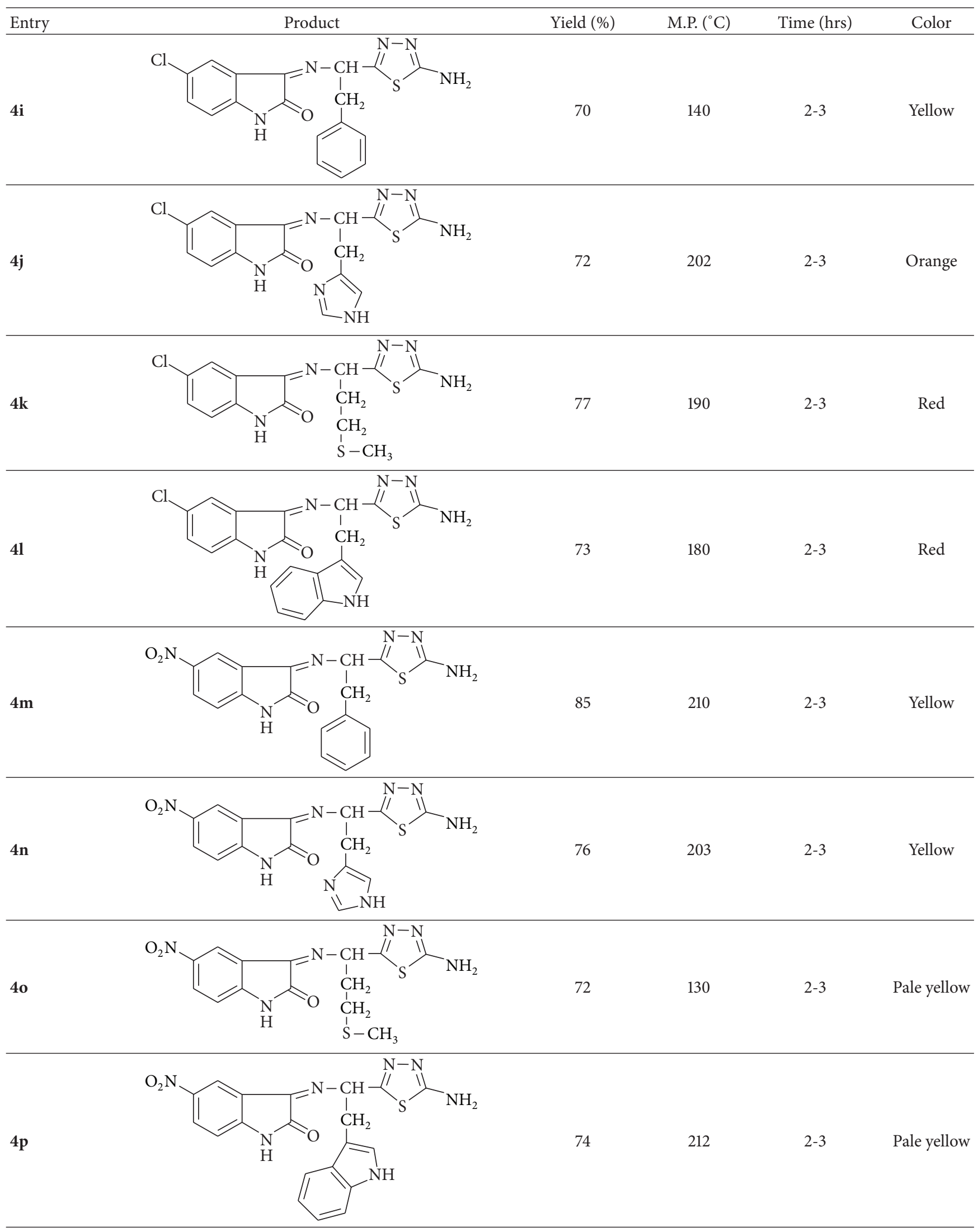




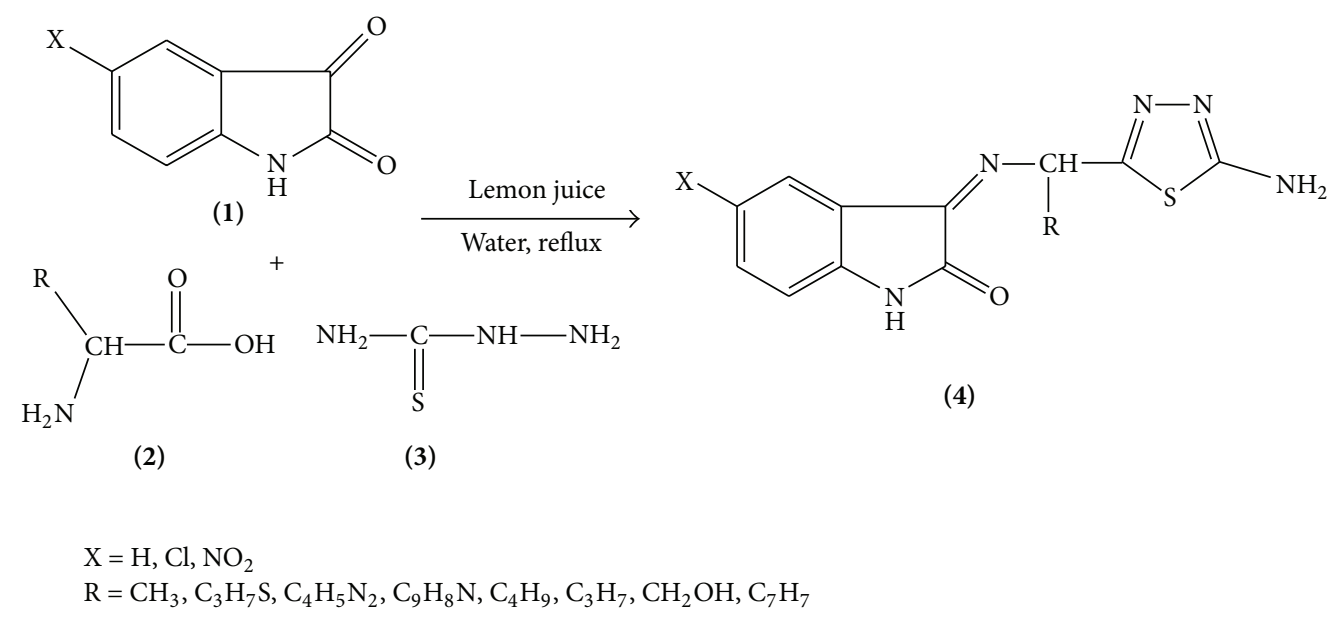

SCHEME 1

The spectral analyses of synthesized compounds have been carried out at SAIF, Punjab University, Chandigarh. Purity of all compounds was checked by TLC using "G" coated glass plates and n-hexane: ethyl acetate $(7: 3)$ as eluent. IR spectra were recorded in $\mathrm{KBr}$ on a Perkin Elmer Infrared RXI FTIR spectrophotometer and ${ }^{1} \mathrm{HNMR}$ spectra were recorded on Bruker Avance II 400 NMR spectrometer using DMSO$\mathrm{d}_{6}$ and $\mathrm{CDCl}_{3}$ as solvent and tetramethylsilane (TMS) as internal reference standard. The obtained products were identified from their spectral $\left({ }^{1} \mathrm{HNMR},{ }^{13} \mathrm{C} \mathrm{NMR}\right.$, and IR) studies.

3.2. General Procedure for Extraction of Lemon Juice. Fresh lemon was cut by using knife and then pieces were pressed manually using domestic presser to extract juice. Then juice was then filtered through cotton/muslin cloth and then through filter paper to remove solid material and to get clear juice which was used as a catalyst.

3.3. Green Route for the Synthesis of 3-[1-(5-Amino-[1,3,4]thiadiazol-2-yl)-substitutedethylimino]-5-substituted-2,3-dih$y$ dro-indol-2-ones $(\mathbf{4 a - p})$. An equimolar mixture of substituted $1 \mathrm{H}$-indole-2,3-diones $(0.012 \mathrm{~mol})$, amino acids (0.012 $\mathrm{mol})$, and thiosemicarbazide in water $(15 \mathrm{~mL}$ containing $1 \mathrm{~mL}$ of ethanol) in the presence of lemon juice $(2 \mathrm{~mL})$ was mixed in round bottom flask and the mixture was refluxed for the time needed to complete the reaction (as monitored by TLC). The initial syrupy reaction mixture solidifies within $2-3 \mathrm{hrs}$. After completion of reaction, mixture was cooled to room temperature and the solid mass obtained was filtered, washed with water, and dried. The desired products were obtained with satisfactory yields without any further purification.

The spectroscopic characterization data of $(\mathbf{4} \mathbf{a}-\mathbf{p})$ are given below.

3-[1-(5-Amino-[1,3,4]thiadiazol-2-yl)-ethylimino]-2,3-dihydro-indol-2-one (4a). m.p. $195^{\circ} \mathrm{C}$; IR (KBr): 3259, 3157, 2923 , $1706,1619,1585,1462,673 \mathrm{~cm}^{-1} ;{ }^{1} \mathrm{H}$ NMR (DMSO-d $\left.{ }_{6}\right): 1.56$ (d, $3 \mathrm{H}, \mathrm{CH}_{3}$ ), 4.59 (q, $\left.1 \mathrm{H}, \mathrm{CH}\right), 7.03-7.67$ (m, $4 \mathrm{H}$, aromatic), 7.22 (s, $2 \mathrm{H}, \mathrm{NH}_{2}$ ), 11.18 (s, $\left.1 \mathrm{H}, \mathrm{N}-\mathrm{H}\right)$ ppm. ${ }^{13} \mathrm{C}-\mathrm{NMR}(400 \mathrm{MHz}$, DMSO): $23.8,51.4,120,122,124,128,132,146.4,162,176$, 178. Anal.calcd for $\mathrm{C}_{12} \mathrm{H}_{11} \mathrm{~N}_{5}$ OS: C, 52.73; H, 4.06; N, 25.62 . Found: C, 52.91; H, 4.09; N, 25.63.

3-[1-(5-Amino-[1, 3,4] thiadiazol-2-yl)-3-methylsulfanyl-propylimino]-2,3-dihydro-indol-2-one (4b). m.p. $170^{\circ} \mathrm{C}$; IR (KBr): $3263,3177,2980,1706,1620,1580,1462,670 \mathrm{~cm}^{-1} ;{ }^{1} \mathrm{H}$ NMR $\left(\mathrm{DMSO}_{6} \mathrm{~d}_{6}\right): 2.09\left(\mathrm{~s}, 3 \mathrm{H}, \mathrm{CH}_{3}\right), 2.31\left(\mathrm{q}, 2 \mathrm{H}, \mathrm{CH}_{2}\right), 2.44(\mathrm{t}$, $2 \mathrm{H}, \mathrm{CH}_{2}$ ), 4.41 (t, 1H, CH), 7.03-7.67 (m, 4H, aromatic), 7.22 (s, $\left.2 \mathrm{H}, \mathrm{NH}_{2}\right), 11.18(\mathrm{~s}, 1 \mathrm{H}, \mathrm{N}-\mathrm{H})$ ppm. ${ }^{13} \mathrm{C}-\mathrm{NMR}(400 \mathrm{MHz}$, DMSO): 17, 30.4, 38.2, 52.4, 122, 124, 126, 128, 132, 146.2, 160, 172, 174. Anal.calcd for $\mathrm{C}_{14} \mathrm{H}_{15} \mathrm{~N}_{5} \mathrm{OS}_{2}$ : C, 50.43; $\mathrm{H}, 4.53 ; \mathrm{N}$, 21.00. Found: C, 50.24; H, 4.56; N, 21.02.

3-[1-(5-Amino- $[1,3,4]$ thiadiazol-2-yl)-2-(1H-imidazol-4-yl)ethylimino]-2,3-dihydro-indol-2-one (4c). m.p. $103^{\circ} \mathrm{C}$; IR (KBr): 3260, 3175, 2986, 1705, 1619, 1585, 1462, $673 \mathrm{~cm}^{-1} ;{ }^{1} \mathrm{H}$ NMR (DMSO-d ${ }_{6}$ ): 3.33 (d, 2H, $\mathrm{CH}_{2}$ ), $4.80(\mathrm{t}, 1 \mathrm{H}, \mathrm{CH}), 6.80$ (d, $1 \mathrm{H}, \mathrm{CH}), 8.73$ (d, 1H, CH), 7.03-7.67 (m, 4H, aromatic), 7.22 (s, 2H, NH ), 11.18 (s, 1H, N-H), 13.4 (dd, 1H, NH) ppm. ${ }^{13} \mathrm{C}-\mathrm{NMR}$ (400 MHz, DMSO): 36, 56, 118, 120, 122.6, 124.8, $128.2,132.2,134.2,136.8,146.8,162.8,174.2,176$. Anal.calcd for $\mathrm{C}_{15} \mathrm{H}_{13} \mathrm{~N}_{7}$ OS: C, 53.09; H, 3.86; N, 28.89. Found: C, 53.27; H, 3.89; N, 28.91.

3-[1-(5-Amino- $[1,3,4]$ thiadiazol-2-yl)-2-(1H-indol-3-yl)-ethylimino]-2,3-dihydro-indol-2-one (4d). m.p. $190^{\circ} \mathrm{C}$; IR (KBr): $3263,3177,2980,1706,1619,1580,1462,670 \mathrm{~cm}^{-1} ;{ }^{1} \mathrm{H}$ NMR $\left(\mathrm{DMSO}_{-} \mathrm{d}_{6}\right): 3.20\left(\mathrm{~d}, 2 \mathrm{H}, \mathrm{CH}_{2}\right), 4.80(\mathrm{t}, 1 \mathrm{H}, \mathrm{CH}), 7.18(\mathrm{~s} 1 \mathrm{H}$, $\mathrm{CH}), 10.85$ (s, 1H, NH), 7.03-7.67 (m, 4H, aromatic), 6.97$7.58\left(\mathrm{~m}, 4 \mathrm{H}\right.$, aromatic), $7.22\left(\mathrm{~s}, 2 \mathrm{H}, \mathrm{NH}_{2}\right), 11.18(\mathrm{~s}, 1 \mathrm{H}, \mathrm{N}-$ H) ppm. ${ }^{13} \mathrm{C}-\mathrm{NMR}(400 \mathrm{MHz}, \mathrm{DMSO}): 36.8,58,110,112$, $118,120.2,122.8,124,128,130.2,132.6,136,146,164.4,176$, 178.2. Anal.calcd for $\mathrm{C}_{20} \mathrm{H}_{16} \mathrm{~N}_{6} \mathrm{OS}$ : C, 61.84; H, 4.15; N, 21.63 . Found: C, 61.66; H, 4.18; N, 21.65.

3-[1-(5-Amino-[1, 3,4]thiadiazol-2-yl)-2-methylbutylimino]2,3-dihydro-indol-2-one (4e). m.p. $170^{\circ} \mathrm{C}$; IR (KBr): 3263, 3086, 2986, 2876, 1733, 1621, 1594, 1462, $673 \mathrm{~cm}^{-1}$; ${ }^{1} \mathrm{H}$ NMR 
$\left(\mathrm{DMSO}_{6} \mathrm{~d}_{6}\right): 0.96\left(\mathrm{t}, 3 \mathrm{H}, \mathrm{CH}_{3}\right), 1.29\left(\mathrm{q}, 2 \mathrm{H}, \mathrm{CH}_{2}\right), 1.06$ (d, $\left.3 \mathrm{H}, \mathrm{CH}_{3}\right), 2.00(\mathrm{~m}, 1 \mathrm{H}, \mathrm{CH}), 4.40(\mathrm{~d}, 1 \mathrm{H}, \mathrm{CH}), 7.03-7.67(\mathrm{~m}$, $4 \mathrm{H}$, aromatic), 7.22 (s, $2 \mathrm{H}, \mathrm{NH}_{2}$ ), 11.18 (s, $\left.1 \mathrm{H}, \mathrm{N}-\mathrm{H}\right)$ ppm. ${ }^{13} \mathrm{C}-\mathrm{NMR}$ (400 MHz, DMSO): 11.6, 15.8, 24.8, 38, 58.2, 120.2, $122.2,124.8,128,132,142.2,162.2,176.2$, 178. Anal.calcd for $\mathrm{C}_{15} \mathrm{H}_{17} \mathrm{~N}_{5} \mathrm{OS}$ : C, 57.12; H, 5.43; N, 22.21. Found: C, 57.32; H, $5.45 ; \mathrm{N}, 22.23$.

3-[1-(5-Amino-[1,3,4] thiadiazol-2-yl)-2-methylpropylimino]2,3-dihydro-indol-2-one (4f). m.p. $190^{\circ} \mathrm{C}$; IR (KBr): 3260, $3150,2877,2803,1695,1620,1580,1465,670 \mathrm{~cm}^{-1} ;{ }^{1} \mathrm{H}$ NMR $\left(\mathrm{DMSO}_{-} \mathrm{d}_{6}\right): 1.01\left(\mathrm{~d}, 3 \mathrm{H}, \mathrm{CH}_{3}\right), 1.01\left(\mathrm{~d}, 3 \mathrm{H}, \mathrm{CH}_{3}\right), 2.2(\mathrm{~m}$, $1 \mathrm{H}, \mathrm{CH}), 4.40$ (d, 1H, CH), 7.03-7.67 (m, 4H, aromatic), 7.22 (s, $2 \mathrm{H}, \mathrm{NH}_{2}$ ), 11.18 (s, 1H, N-H) ppm. ${ }^{13} \mathrm{C}-\mathrm{NMR}(400 \mathrm{MHz}$, DMSO): 18.2, 36.2, 78.6, 110, 126, 128, 130, 142, 143, 162.8, 176, 178.2. Anal.calcd for $\mathrm{C}_{14} \mathrm{H}_{15} \mathrm{~N}_{5}$ OS: C, 55.80; H, 5.02; N, 23.24. Found: C, 55.62; H, 5.04; N, 23.25.

3-[1-(5-Amino-[1,3,4]thiadiazol-2-yl)-2-hydroxyethylimino]2,3-dihydro-indol-2-one (4g). m.p. $98^{\circ} \mathrm{C}$; IR (KBr): 3465, $3263,3177,2980,1706,1619,1580,1462,673 \mathrm{~cm}^{-1} ;{ }^{1} \mathrm{H}$ NMR $\left(\mathrm{DMSO}_{-} \mathrm{d}_{6}\right): 4.30\left(\mathrm{~d}, 2 \mathrm{H}, \mathrm{CH}_{2}\right), 4.50(\mathrm{t}, 1 \mathrm{H}, \mathrm{CH}), 4.78(\mathrm{~s}, 1 \mathrm{H}$, $\mathrm{OH}), 7.03-7.67$ (m, 4H, aromatic), $7.22\left(\mathrm{~s}, 2 \mathrm{H}, \mathrm{NH}_{2}\right), 11.18$ (s, 1H, N-H) ppm. ${ }^{13} \mathrm{C}-\mathrm{NMR}(400 \mathrm{MHz}, \mathrm{DMSO}): 51.4,68$, $120,122,124,128,132,146.2,162.8,176.2,178.8$. Anal.calcd for $\mathrm{C}_{12} \mathrm{H}_{11} \mathrm{~N}_{5} \mathrm{O}_{2} \mathrm{~S}$ : C, 49.82; H, 3.83; N, 24.21. Found: C, 49.63; $\mathrm{H}, 3.85$; N, 24.22.

3-[1-(5-Amino-[1, 3,4]thiadiazol-2-yl)-2-phenyl-ethylimino]2,3-dihydro-indol-2-one (4h). m.p. $140^{\circ} \mathrm{C}$; IR (KBr): 3265, $3175,2986,1705,1620,1580,1465,670 \mathrm{~cm}^{-1} ;{ }^{1} \mathrm{H}$ NMR $\left(\mathrm{DMSO}_{-} \mathrm{d}_{6}\right): 3.33\left(\mathrm{~d}, 2 \mathrm{H}, \mathrm{CH}_{2}\right), 4.80(\mathrm{t}, 1 \mathrm{H}, \mathrm{CH}), 7.12-7.26$ (m, 5H, aromatic), 7.03-7.67 (m, $4 \mathrm{H}$, aromatic), $7.22(\mathrm{~s}, 2 \mathrm{H}$, $\mathrm{NH}_{2}$ ), 11.18 (s, 1H, N-H) ppm. ${ }^{13} \mathrm{C}-\mathrm{NMR}$ (400 MHz, DMSO): 42.8, 56.4, 120, 122, 124.2, 128.8, 132, 138, 146.2, 162.8, 176.2, 178. Anal.calcd for $\mathrm{C}_{18} \mathrm{H}_{15} \mathrm{~N}_{5}$ OS: C, 61.87; H, 4.33; N, 20.04. Found: C, 61.68; H, 4.35; N, 20.06.

3-[1-(5-Amino-[1, 3,4]thiadiazol-2-yl)-2-phenylethylimino]-5chloro-2,3-dihydro-indol-2-one (4i). m.p. $140^{\circ} \mathrm{C}$; IR ( $\left.\mathrm{KBr}\right)$ : $3263,3177,2980,1706,1619,1580,1462,695,670 \mathrm{~cm}^{-1} ;{ }^{1} \mathrm{H}$ NMR (DMSO-d ${ }_{6}$ ): 3.33 (d, 2H, $\mathrm{CH}_{2}$ ), 4.80 (t, 1H, CH), 7.12$7.26(\mathrm{~m}, 5 \mathrm{H}$, aromatic), 7.28-7.61 ( $\mathrm{m}, 3 \mathrm{H}$, aromatic), 7.22 (s, $2 \mathrm{H}, \mathrm{NH}_{2}$ ), 11.18 (s, $\left.1 \mathrm{H}, \mathrm{N}-\mathrm{H}\right)$ ppm. ${ }^{13} \mathrm{C}-\mathrm{NMR}(400 \mathrm{MHz}$, DMSO): 42, 56, 120.2, 122.4, 124, 128, 132, 144, 162, 176, 178.8 . Anal.calcd for $\mathrm{C}_{18} \mathrm{H}_{14} \mathrm{ClN}_{5} \mathrm{OS}$ : C, 56.32; H, 3.68; N, 18.24. Found: C, 56.51; H, 3.66; N, 18.25.

3-[1-(5-Amino- $[1,3,4]$ thiadiazol-2-yl)-2-(1H-imidazol-4yl)-ethylimino]-5-chloro-2,3-dihydro-indol-2-one (4j). m.p. $202^{\circ} \mathrm{C}$; IR (KBr): $3265,3170,2985,1705,1619,1580,1462,698$, $670 \mathrm{~cm}^{-1} ;{ }^{1} \mathrm{H}$ NMR (DMSO-d $\left.\mathrm{D}_{6}\right): 3.33\left(\mathrm{~d}, 2 \mathrm{H}, \mathrm{CH}_{2}\right), 4.80(\mathrm{t}$, $1 \mathrm{H}, \mathrm{CH}), 6.80(\mathrm{~d}, 1 \mathrm{H}, \mathrm{CH}), 8.73(\mathrm{~d}, 1 \mathrm{H}, \mathrm{CH}), 7.28-7.61(\mathrm{~m}$, $3 \mathrm{H}$, aromatic), 7.22 (s, 2H, $\mathrm{NH}_{2}$ ), 11.18 (s, 1H, N-H), 13.4 (dd, 1H, NH) ppm. ${ }^{13} \mathrm{C}-\mathrm{NMR}(400 \mathrm{MHz}, \mathrm{DMSO}): 38,56.8$, $120,122.2,124,128.6,132,136,162.2,176.4,178$. Anal.calcd for $\mathrm{C}_{15} \mathrm{H}_{12} \mathrm{ClN}_{7} \mathrm{OS}$ : C, 48.19; H, 3.24; N, 26.23. Found: C, 48.38; $\mathrm{H}, 3.26 ; \mathrm{N}, 26.24$.
3-[1-(5-Amino-[1, 3,4] thiadiazol-2-yl)-3-methylsulfanyl-propylimino]-5-chloro-2,3-dihydro-indol-2-one (4k). m.p. $190^{\circ} \mathrm{C}$; IR (KBr): 3263, 3177, 2980, 1706, 1620, 1580, 1465, 690, $673 \mathrm{~cm}^{-1} ;{ }^{1} \mathrm{H}$ NMR (DMSO-d $): 2.09\left(\mathrm{~s}, 3 \mathrm{H}, \mathrm{CH}_{3}\right), 2.31(\mathrm{q}$, $\left.2 \mathrm{H}, \mathrm{CH}_{2}\right), 2.44\left(\mathrm{t}, 2 \mathrm{H}, \mathrm{CH}_{2}\right), 4.41(\mathrm{t}, 1 \mathrm{H}, \mathrm{CH}), 7.28-7.61(\mathrm{~m}$, $3 \mathrm{H}$, aromatic), 7.22 (s, $\left.2 \mathrm{H}, \mathrm{NH}_{2}\right), 11.18(\mathrm{~s}, 1 \mathrm{H}, \mathrm{N}-\mathrm{H}) \mathrm{ppm} .{ }^{13} \mathrm{C}-$ NMR (400 MHz, DMSO): 18, 30.4, 38, 54.4, 120.2, 122.8, 124.4, $128,132,144,162,176$ and 178. Anal.calcd for $\mathrm{C}_{14} \mathrm{H}_{14} \mathrm{ClN}_{5} \mathrm{OS}_{2}$ : C, 45.71; H, 3.84; N, 19.04. Found: C, 45.52; H, 3.86; N, 19.05.

3-[1-(5-Amino- $[1,3,4]$ thiadiazol-2-yl)-2-(1H-indol-3-yl)-ethylimino]-5-chloro-2,3-dihydro-indol-2-one (4l). m.p. $180^{\circ} \mathrm{C}$; IR (KBr): 3265, 3175, 2986, 1705, 1619, 1580, 1462, 695, $670 \mathrm{~cm}^{-1}$; ${ }^{1} \mathrm{H}$ NMR (DMSO-d $\left.{ }_{6}\right): 3.20\left(\mathrm{~d}, 2 \mathrm{H}, \mathrm{CH}_{2}\right), 4.80(\mathrm{t}, 1 \mathrm{H}, \mathrm{CH})$, 7.18 (s 1H, CH), 10.85 (s, 1H, NH), 7.28-7.61 (m, 3H, aromatic), 6.97-7.58 (m, 4H, aromatic), $7.22\left(\mathrm{~s}, 2 \mathrm{H}, \mathrm{NH}_{2}\right), 11.18(\mathrm{~s}, 1 \mathrm{H}, \mathrm{N}-$ H) ppm. ${ }^{13}$ C-NMR (400 MHz, DMSO): 36, 58.4, 110, 112, 120, $122,124,128.2,132,136,144.4,162.8,176.2,178.2$. Anal.calcd for $\mathrm{C}_{20} \mathrm{H}_{15} \mathrm{ClN}_{6} \mathrm{OS}$ : C, 56.80; H, 3.58; N, 19.87. Found: C, 56.61; H, $3.55 ; \mathrm{N}, 19.89$.

3-[1-(5-Amino-[1,3,4]thiadiazol-2-yl)-2-phenyl-ethylimino]5-nitro-2,3-dihydro-indol-2-one (4m). m.p. $210^{\circ} \mathrm{C}$; IR ( $\left.\mathrm{KBr}\right)$ : $3263,3177,2980,1706,1615,1580,1462,1362,670 \mathrm{~cm}^{-1} ;{ }^{1} \mathrm{H}$ NMR (DMSO-d $\left.)_{6}\right): 3.33\left(\mathrm{~d}, 2 \mathrm{H}, \mathrm{CH}_{2}\right), 4.80(\mathrm{t}, 1 \mathrm{H}, \mathrm{CH})$, $7.12-7.26$ (m, 5H, aromatic), 7.93-8.53 (m, 3H, aromatic), 7.22 (s, $2 \mathrm{H}, \mathrm{NH}_{2}$ ), 11.18 (s, $\left.1 \mathrm{H}, \mathrm{N}-\mathrm{H}\right)$ ppm. ${ }^{13} \mathrm{C}-\mathrm{NMR}(400 \mathrm{MHz}$, DMSO): 42, 56.4, 120, 122, 124, 128, 132, 144, 152, 162, 176, 178. Anal.calcd for $\mathrm{C}_{18} \mathrm{H}_{14} \mathrm{~N}_{6} \mathrm{O}_{3} \mathrm{~S}$ : C, 54.81; H, 3.58; N, 21.31. Found: C, 54.62; H, 3.56; N, 21.34.

3-[1-(5-Amino-[1, 3,4] thiadiazol-2-yl)-2-(1H-imidazol-4-yl)ethylimino]-5-nitro-2,3-dihydro-indol-2-one (4n). m.p. $203^{\circ} \mathrm{C}$; IR (KBr): 3265, 3175, 2986, 1705, 1619, 1585, 1460, 1365, $672 \mathrm{~cm}^{-1} ;{ }^{1} \mathrm{H}$ NMR (DMSO-d $)$ ): $3.33\left(\mathrm{~d}, 2 \mathrm{H}, \mathrm{CH}_{2}\right), 4.80(\mathrm{t}$, $1 \mathrm{H}, \mathrm{CH}), 6.80(\mathrm{~d}, 1 \mathrm{H}, \mathrm{CH}), 8.73(\mathrm{~d}, 1 \mathrm{H}, \mathrm{CH}), 7.93-8.53(\mathrm{~m}$, $3 \mathrm{H}$, aromatic), 7.22 (s, 2H, $\mathrm{NH}_{2}$ ), 11.18 (s, $\left.1 \mathrm{H}, \mathrm{N}-\mathrm{H}\right), 13.4$ (dd, 1H, NH) ppm. ${ }^{13} \mathrm{C}-\mathrm{NMR}$ (400 MHz, DMSO): 37, 56, $118,120.2,122.8,124.6,128.2,132.2,144.4,152.2,162.8,176.2$, 178.4. Anal.calcd for $\mathrm{C}_{15} \mathrm{H}_{12} \mathrm{~N}_{8} \mathrm{O}_{3} \mathrm{~S}$ : C, 46.87; H, 3.15; N, 29.15. Found: C, 46.68; H, 3.17; N, 29.17.

3-[1-(5-Amino-[1, 3,4]thiadiazol-2-yl)-3-methylsulfanyl-propylimino]-5-nitro-2,3-dihydro-indol-2-one (4o). m.p. $130^{\circ} \mathrm{C}$; IR (KBr): 3260, 3170, 2985, 1702, 1615, 1580, 1462, 1362, $670 \mathrm{~cm}^{-1} ;{ }^{1} \mathrm{H}$ NMR (DMSO-d $): 2.09\left(\mathrm{~s}, 3 \mathrm{H}, \mathrm{CH}_{3}\right), 2.31(\mathrm{q}$, $\left.2 \mathrm{H}, \mathrm{CH}_{2}\right), 2.44\left(\mathrm{t}, 2 \mathrm{H}, \mathrm{CH}_{2}\right), 4.41(\mathrm{t}, 1 \mathrm{H}, \mathrm{CH}), 7.93-8.53(\mathrm{~m}$, $3 \mathrm{H}$, aromatic), 7.22 (s, 2H, $\mathrm{NH}_{2}$ ), 11.18 (s, 1H, N-H) ppm. ${ }^{13} \mathrm{C}-\mathrm{NMR}$ (400 MHz, DMSO): 18, 30, 38, 54, 122.2, 124.8, $128.4,134.2,144.8,152.6,162.2,176.4,178.2$. Anal.calcd for $\mathrm{C}_{14} \mathrm{H}_{14} \mathrm{~N}_{6} \mathrm{O}_{3} \mathrm{~S}_{2}$ : C, 44.43; H, 3.73; N, 22.21. Found: C, 44.62; $\mathrm{H}, 3.71 ; \mathrm{N}, 22.23$.

3-[1-(5-Amino-[1,3,4]thiadiazol-2-yl)-2-(1H-indol-3-yl)-ethylimino]-5-nitro-2,3-dihydro-indol-2-one (4p). m.p. $212^{\circ} \mathrm{C}$; IR (KBr): 3263, 3177, 2980, 1706, 1620, 1585, 1460, 1362, $675 \mathrm{~cm}^{-1}$; ${ }^{1} \mathrm{H}$ NMR (DMSO-d $\left.{ }_{6}\right): 3.20\left(\mathrm{~d}, 2 \mathrm{H}, \mathrm{CH}_{2}\right), 4.80(\mathrm{t}, 1 \mathrm{H}$, $\mathrm{CH}), 7.18$ (s $1 \mathrm{H}, \mathrm{CH}), 10.85$ (s, $1 \mathrm{H}, \mathrm{NH}), 7.93-8.53(\mathrm{~m}, 3 \mathrm{H}$, aromatic), 6.97-7.58 (m, $4 \mathrm{H}$, aromatic), $7.22\left(\mathrm{~s}, 2 \mathrm{H}, \mathrm{NH}_{2}\right)$, 
11.18 (s, 1H, N-H) ppm. ${ }^{13} \mathrm{C}-\mathrm{NMR}$ (400 MHz, DMSO): 36, $58.4,110,118,120.2,122,124,128,132,144,152,162,176.8$, 178. Anal.calcd for $\mathrm{C}_{20} \mathrm{H}_{15} \mathrm{~N}_{7} \mathrm{O}_{3} \mathrm{~S}$ : C, 55.42; H, 3.49; N, 22.62. Found: C, 55.23; H, 3.47; N, 22.63.

\section{Antimicrobial Activity}

4.1. Antibacterial Activity. All the synthesized compounds were tested for in vitro antibacterial activity by twofold serial dilution assay [46] against the reference compound streptomycin. The minimal inhibitory concentration (MIC) is shown in Tables 2 and 4. During MIC determination by twofold serial dilution method, compounds $\mathbf{4 g}, \mathbf{4 j}$, and $\mathbf{4 m}$ exhibit good activities against all bacterial strains at a MIC value of $1000 \mu \mathrm{g} / \mathrm{mL}$. Admirable activities against $M$. luteus and $E$. coli are displayed by compounds $4 \mathbf{d}, \mathbf{4 e}, \mathbf{4 g}, \mathbf{4 h}, \mathbf{4 i}, \mathbf{4 j}$, 4l, and $4 \mathbf{o}$ at a MIC value of $500 \mu \mathrm{g} / \mathrm{mL}$. Compounds, $4 \mathbf{a}, \mathbf{4 e}$, 4o, and $\mathbf{4 p}$ display higher activities against $S$. aureus, at a MIC value of $1000 \mu \mathrm{g} / \mathrm{mL}$.

4.2. Antifungal Activity. The antifungal activity of the compounds was also studied with Aspergillus sp., Penicillium sp., Alternaria brassicicola, Chaetomium orium, and Lycopodium $s p$. fungi. The results are summarized in Tables 2 and 3. It has been observed that all synthesized chemical compounds showed lesser antifungal activity as compared to antibacterial activity. On MIC determination, compounds $\mathbf{4 h}, \mathbf{4 i}, \mathbf{4 k}$, and 4m showed excellent antimicrobial activities at a MIC value of $1000 \mu \mathrm{g} / \mathrm{mL}$. Modest activities are displayed by compound 4n against Aspergillus sp. and Chaetomium orium at a MIC value of $>1000 \mu \mathrm{g} / \mathrm{mL}$, whereas compound $\mathbf{4 a}, \mathbf{4 c}$, and $\mathbf{4 g}$ exhibit mild activities against Aspergillus sp. and Lycopodium $s p$. at a MIC value of $1000 \mu \mathrm{g} / \mathrm{mL}$.

Determination of Minimal Inhibitory Concentrations (MIC). Minimum inhibitory concentration was determined by twofold serial dilution method [46]. A series of test tubes were prepared containing the same volume of media inoculated with the test organism. Drug was added to the tubes in a stepwise dilution by a factor of 2 (twofold serial dilution); that is, the concentration of drug in the first tube is $1000 \mu \mathrm{g} / \mathrm{mL}$, in the second tube it will be $500 \mu \mathrm{g} / \mathrm{mL}$, and in the third it will be $250 \mu \mathrm{g} / \mathrm{mL}$. Cultures were incubated at $24 \mathrm{hrs}$ for bacteria at $37^{\circ} \mathrm{C}$ and $48 \mathrm{hrs}$ for fungi at $30^{\circ} \mathrm{C}$. One tube was left without drug only solvent to serve as a positive control for the growth of the organism. After incubation, tubes were examined for microbial growth and the minimum inhibitory concentrations (the minimum concentration of each treatment that inhibits microbial growth after the incubation period) were measured. Tubes are inspected visually to determine the growth of the organism indicated by turbidity and simultaneously streak on Petri dish containing nutrient agar medium; after incubation ( 24 hours for bacteria and $48 \mathrm{hrs}$ for fungal strains) microbial growth were measured.

From the screening results, it is observed that the presence of electron withdrawing groups like $\mathrm{Cl}$ and $\mathrm{NO}_{2}$ group at 5th position of indole ring exhibits significant activity in comparison to unsubstituted compounds. However other synthesized compounds of the series also exhibit moderate to significant activity against the microorganisms as mentioned above.

4.3. Evaluation of Cytotoxic Effects. The uncontrolled growth of cells in the body, started due to certain stimuli, lays the foundation of cancer. Anticancer drugs either kill cancer cells or modify their growth. Among the heterocyclic compounds, five membered heterocyclic moieties fused with aromatic ring system with various heteroatoms like $\mathrm{N}, \mathrm{S}$, and $\mathrm{O}$ are potential anticancer agents [47]. Synthesis of nitrogen containing heterocyclic compounds has been a subject of great interest due to the wide application in agrochemical and pharmaceutical fields. Heterocycles like indole, pyrimidine, pyridine, quinoline, and so forth are an integral part of a huge number of natural and synthetic compounds and play important roles in the biological systems. For developing the suitable leads for anticancer drugs, introduction of appropriate substituents at C-3 of indole is required [48]. It has also been observed that Schiff bases also play a major role in developing cytotoxic drugs $[49,50]$. Hence, an attempt has been made to synthesize Schiff bases incorporating indole moiety and the synthesized compounds $\mathbf{4 a}, \mathbf{4 e}, \mathbf{4 f}$, and $\mathbf{4 h}$ were evaluated [51] for their cytotoxic effects against Human colon cancer cell line Colo205. Percent growth was calculated on a plate-by-plate basis for test wells relative to control wells.

Percent growth was expressed as the ratio of average absorbance of the test well to the average absorbance of the control wells $* 100$.

Using the six-absorbance measurements [time zero $(\mathrm{Tz})$, control growth (C), and test growth in the presence of drug at the four concentration levels $(\mathrm{Ti})]$, the percentage growth was calculated at each of the drug concentration levels.

Percentage growth inhibition was calculated as

$[(\mathrm{Ti}-\mathrm{Tz}) /(\mathrm{C}-\mathrm{Tz})] \times 100$ for concentrations for which $\mathrm{Ti} \geq \mathrm{Tz}(\mathrm{Ti}-\mathrm{Tz})$ positive or zero,

$[(\mathrm{Ti}-\mathrm{Tz}) / \mathrm{Tz}] \times 100$ for concentrations for which $\mathrm{Ti}<$ Tz. $(\mathrm{Ti}-\mathrm{Tz})$ negative.

The dose response parameters were calculated for each test article. Growth inhibition of 50\% (GI50) was calculated from $[(\mathrm{Ti}-\mathrm{Tz}) /(\mathrm{C}-\mathrm{Tz})] \times 100=50$, which is the drug concentration resulting in a $50 \%$ reduction in the net protein increase (as measured by SRB staining) in control cells during the drug incubation. The drug concentration resulting in total growth inhibition (TGI) was calculated from $\mathrm{Ti}=\mathrm{Tz}$. The LC50 (concentration of drug resulting in a $50 \%$ reduction in the measured protein at the end of the drug treatment as compared to that at the beginning) indicating a net loss of cells following treatment is calculated from

$$
\left[\frac{(\mathrm{Ti}-\mathrm{Tz})}{\mathrm{Tz}}\right] \times 100=-50 \text {. }
$$

Values were calculated for each of these three parameters if the level of activity was reached; however, if the effect was not reached or was exceeded, the values for that parameter were expressed as greater or less than the maximum or minimum concentration tested. The data of anticancer 


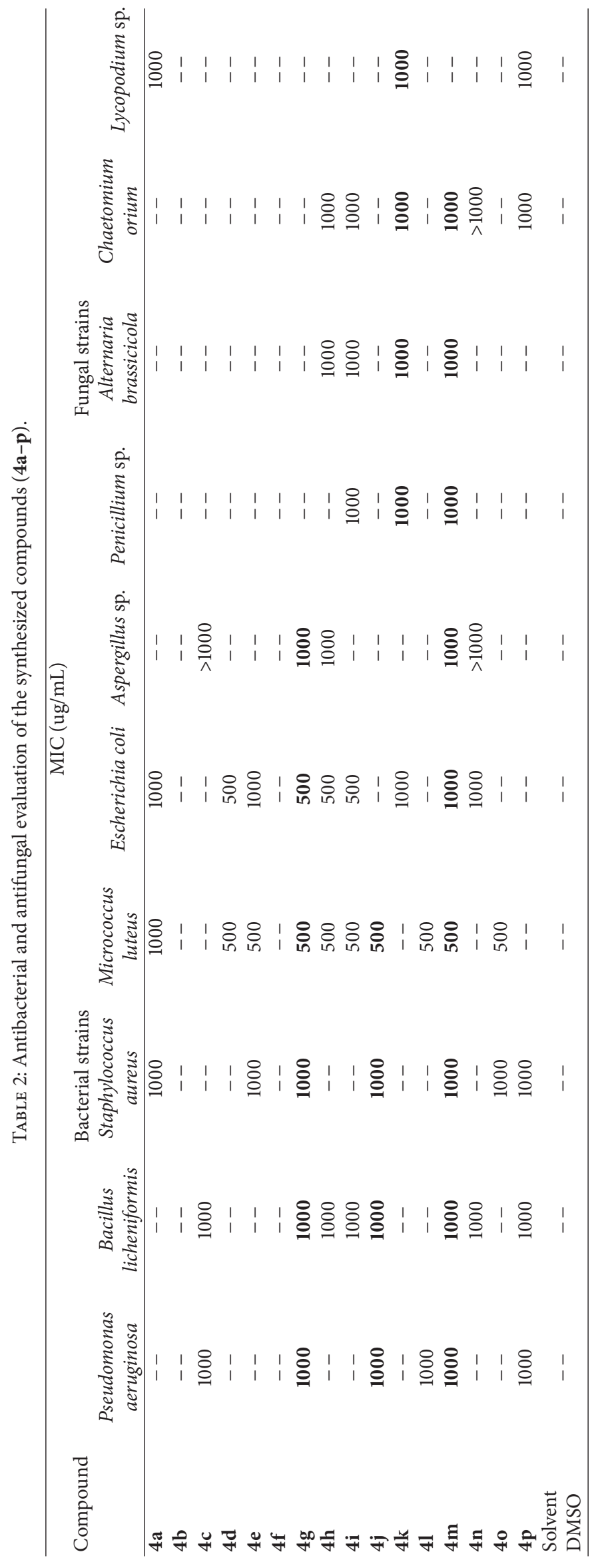


TABLE 3: Minimum inhibitory concentration for fungi.

\begin{tabular}{|c|c|c|c|c|c|c|c|c|c|c|c|c|c|c|c|}
\hline \multirow{3}{*}{ Compound } & \multicolumn{15}{|c|}{ MIC (ug/mL) } \\
\hline & \multicolumn{3}{|c|}{ Aspergillus sp. } & \multicolumn{3}{|c|}{ Penicillium sp. } & \multicolumn{3}{|c|}{ Alternaria brassicicola } & \multicolumn{3}{|c|}{ Chaetomium orium } & \multicolumn{3}{|c|}{ Lycopodium sp. } \\
\hline & 250 & 500 & 1000 & 250 & 500 & 1000 & 250 & 500 & 1000 & 250 & 500 & 1000 & 250 & 500 & 1000 \\
\hline $4 a$ & + & + & + & + & + & + & + & + & + & + & + & + & + & - & $--^{*}$ \\
\hline $4 b$ & + & + & + & + & + & + & + & + & + & + & + & + & + & + & + \\
\hline $4 c$ & + & + & $--^{*}$ & + & + & + & + & + & + & + & + & + & + & + & + \\
\hline 4d & + & + & + & + & + & + & + & + & + & + & + & + & + & + & + \\
\hline $4 e$ & + & + & + & + & + & + & + & + & + & + & + & + & + & + & + \\
\hline $4 f$ & + & + & + & + & + & + & + & + & + & + & + & + & + & + & + \\
\hline $4 g$ & + & + & - & + & + & + & + & + & + & + & + & + & - & + & + \\
\hline $4 h$ & + & + & $--^{*}$ & + & + & + & + & + & $--^{*}$ & + & + & $--^{*}$ & + & + & + \\
\hline $4 i$ & + & + & + & + & + & $--^{*}$ & + & + & $--^{*}$ & + & + & $--^{*}$ & + & + & + \\
\hline $4 j$ & + & + & + & + & + & + & + & + & + & + & + & + & + & + & + \\
\hline $4 \mathrm{k}$ & + & + & + & + & + & $--^{*}$ & + & + & $--^{*}$ & + & + & $--^{*}$ & + & + & $--^{*}$ \\
\hline 41 & + & + & $--^{*}$ & + & + & + & + & + & + & + & + & - & + & + & + \\
\hline $4 m$ & + & + & $--^{*}$ & + & + & $--^{*}$ & + & + & - & + & + & $--^{*}$ & + & + & - \\
\hline $4 n$ & + & + & $--^{*}$ & + & + & + & + & + & - & + & + & $--^{*}$ & + & + & - \\
\hline 40 & + & + & + & + & + & + & + & + & - & + & + & + & + & + & - \\
\hline $4 p$ & + & + & + & + & + & + & + & + & - & + & + & $--^{*}$ & + & + & $--^{*}$ \\
\hline Solvent DMSO & + & + & + & + & + & + & + & + & + & + & + & + & + & + & + \\
\hline Itraconazole & & --- & & & -- & & & & & & - & & & -- & \\
\hline
\end{tabular}

${ }^{*}$ Moderately active (zone of inhibition $8-10 \mathrm{~mm}$ ).

TABLE 4: Minimum inhibitory concentration for bacteria.

\begin{tabular}{|c|c|c|c|c|c|c|c|c|c|c|c|c|c|c|c|}
\hline \multirow{3}{*}{ Compound } & \multicolumn{15}{|c|}{ MIC (ug/mL) } \\
\hline & \multicolumn{3}{|c|}{ Pseudomonas aeruginosa } & \multicolumn{3}{|c|}{ Bacillus licheniformis } & \multicolumn{3}{|c|}{ Staphylococcus aureus } & \multicolumn{3}{|c|}{ Micrococcus luteus } & \multicolumn{3}{|c|}{ Escherichia coli } \\
\hline & 250 & 500 & 1000 & 250 & 500 & 1000 & 250 & 500 & 1000 & 250 & 500 & 1000 & 250 & 500 & 1000 \\
\hline $4 a$ & + & + & + & + & + & + & + & - & $--^{*}$ & + & - & $--^{*}$ & + & - & $--^{*}$ \\
\hline $4 b$ & + & + & + & + & + & + & + & + & + & + & + & + & + & + & + \\
\hline $4 c$ & + & - & $--^{*}$ & + & - & $--^{*}$ & + & + & + & + & + & + & + & - & $--^{*}$ \\
\hline $4 d$ & + & + & + & + & + & + & + & + & + & + & $--^{*}$ & $--^{*}$ & + & $--^{*}$ & $--^{*}$ \\
\hline $4 e$ & + & + & + & + & + & + & + & - & $--^{*}$ & + & $--^{*}$ & $--^{*}$ & + & - & $--^{*}$ \\
\hline $4 f$ & + & + & + & + & + & + & + & + & + & + & + & + & + & + & + \\
\hline $4 \mathrm{~g}$ & + & - & $--^{*}$ & + & - & $--^{*}$ & + & - & $--^{*}$ & + & $--^{*}$ & $--^{*}$ & - & $--^{*}$ & $--^{*}$ \\
\hline $4 h$ & + & + & + & + & - & $--^{*}$ & + & + & + & - & $--^{*}$ & $--^{*}$ & + & $--^{*}$ & $--^{*}$ \\
\hline $4 i$ & + & + & + & + & - & $--^{*}$ & + & + & + & - & $--^{*}$ & $--^{*}$ & - & $--^{*}$ & $--^{*}$ \\
\hline $4 j$ & + & - & $--^{*}$ & + & + & $--^{*}$ & + & + & $--^{*}$ & - & $--^{*}$ & $--^{*}$ & + & $--^{*}$ & $--^{*}$ \\
\hline $4 k$ & + & + & + & + & + & + & + & + & + & + & + & + & + & - & $--^{*}$ \\
\hline 41 & + & + & $--^{*}$ & + & + & + & + & + & + & + & $--^{*}$ & $--^{*}$ & + & + & + \\
\hline $4 \mathrm{~m}$ & + & - & $--^{*}$ & + & + & $--^{*}$ & + & - & -- & + & + & + & + & - & $--^{*}$ \\
\hline $4 n$ & + & + & - & + & + & $--^{*}$ & + & + & + & + & $--^{*}$ & $--^{*}$ & + & + & + \\
\hline 40 & + & + & - & + & + & + & + & - & $--^{*}$ & + & $--^{*}$ & $--^{*}$ & + & + & $--^{*}$ \\
\hline $4 p$ & + & - & $--^{*}$ & + & + & $--^{*}$ & + & - & $--^{*}$ & + & + & + & + & + & + \\
\hline Solvent DMSO & + & + & + & + & + & + & + & + & + & + & + & + & + & + & + \\
\hline Streptomycin & \multicolumn{3}{|c|}{----} & \multicolumn{3}{|c|}{+} & \multicolumn{3}{|c|}{+} & \multicolumn{3}{|c|}{+} & \multicolumn{3}{|c|}{----} \\
\hline Gentamycin & \multicolumn{3}{|c|}{---} & \multicolumn{3}{|c|}{---} & \multicolumn{3}{|c|}{----} & \multicolumn{3}{|c|}{----} & \multicolumn{3}{|c|}{----} \\
\hline
\end{tabular}

+ : inactive (zone of inhibition $<6 \mathrm{~mm}$ ); - slightly active (zone of inhibition $6-8 \mathrm{~mm}$ ); --: moderately active (zone of inhibition $8-10 \mathrm{~mm}$ ); - - - -: highly active (zone of inhibition $>10 \mathrm{~mm}$ ); filled part showing MIC for particular organism. 
TABLE 5: In vitro cytotoxic effects of amino acid Schiff bases against human colon cancer cell line Colo205.

(a)

Human colon cancer cell line Colo205

$\%$ control growth

Entry

Molar drug concentration

\begin{tabular}{lccccccccccccccccc} 
& \multicolumn{3}{c}{ Experiment 1 } & \multicolumn{4}{c}{ Experiment 2 } & \multicolumn{4}{c}{ Experiment 3 } & \multicolumn{3}{c}{ Average values } \\
& $10^{-6} \mathrm{M}$ & $10^{-5} \mathrm{M}$ & $10^{-4} \mathrm{M}$ & $10^{-3} \mathrm{M}$ & $10^{-7} \mathrm{M}$ & $10^{-6} \mathrm{M}$ & $10^{-5} \mathrm{M}$ & $10^{-4} \mathrm{M}$ & $10^{-7} \mathrm{M}$ & $10^{-6} \mathrm{M}$ & $10^{-5} \mathrm{M}$ & $10^{-4} \mathrm{M}$ & $10^{-7} \mathrm{M}$ & $10^{-6} \mathrm{M}$ & $10^{-5} \mathrm{M}$ & $10^{-4} \mathrm{M}$ \\
\hline 4a & 98.3 & 91.4 & 91.2 & 29.4 & 96.3 & 93.2 & 91.8 & 28.9 & 90.4 & 94.9 & 83.6 & 22.9 & 95.0 & 93.2 & 88.9 & 27.0 \\
4e & 100.0 & 100.0 & 100.0 & 23.0 & 100.0 & 100.0 & 100.0 & 23.7 & 100.0 & 100.0 & 100.0 & 23.8 & 100.0 & 100.0 & 100.0 & 23.5 \\
4f & 100.0 & 98.0 & 96.3 & 39.7 & 100.0 & 96.3 & 95.1 & 38.2 & 100.0 & 100.0 & 100.0 & 43.4 & 100.0 & 98.1 & 97.1 & 40.4 \\
4h & 100.0 & 100.0 & 93.1 & -25.0 & 100.0 & 99.4 & 91.5 & -20.5 & 100.0 & 97.9 & 88.1 & -28.3 & 100.0 & 99.1 & 90.9 & -24.6 \\
ADR & 73.4 & 21.7 & -64.1 & -74.7 & 71.1 & 23.3 & -70.2 & -73.5 & 67.5 & 14.1 & -66.1 & -76.3 & 70.7 & 19.7 & -66.8 & -74.8 \\
\hline
\end{tabular}

(b)

\begin{tabular}{lccc}
\hline & \multicolumn{2}{c}{$\begin{array}{c}\text { Human colon cancer cell line Colo205 } \\
\text { Molar drug concentration calculated from graph }\end{array}$} \\
Colo205 & LC50 & TGI & GI50 \\
\hline 4a & $>100$ & $>100$ & 67.2 \\
4e & $>100$ & $>100$ & 68.0 \\
4f & $>100$ & $>100$ & 85.4 \\
4h & $>100$ & 81.6 & 41.8 \\
ADR & 67.7 & 15.3 & $<\mathbf{1 0}$ \\
\hline
\end{tabular}

activity in terms of \% control growth at different molar drug concentrations are shown in Table 4. Unfortunately, the evaluated compounds were not found to be active against human colon cancer cell line. Growth curve is presented in Figure 1.

\subsubsection{Definitions and Notes}

GI50: growth inhibition of 50\% (GI50) is calculated from $[(\mathrm{Ti}-\mathrm{Tz}) /(\mathrm{C}-\mathrm{Tz})] \times 100=50$, drug concentration resulting in a $50 \%$ reduction in the net protein increase;

TGI: drug concentration resulting in total growth inhibition (TGI) will be calculated from $\mathrm{Ti}=\mathrm{Tz}$;

LC50: concentration of drug resulting in a 50\% reduction in the measured protein at the end of the drug treatment (as compared to that at the beginning) indicating a net loss of $50 \%$ cells following treatment is calculated from $[(\mathrm{Ti}-\mathrm{Tz}) / \mathrm{Tz}] \times 100=-50$;

ADR: Adriamycin (doxorubicin) is positive control compound;

GI50 value of $\leq 10^{-6}$ (i.e., $1 \mu$ mole) or $\leq 10 \mu \mathrm{g} / \mathrm{mL}$ is considered to demonstrate activity in case of pure compounds.

For extracts, GI50 value $\leq 20 \mu \mathrm{g} / \mathrm{mL}$ is considered to demonstrate activity.

The bold highlighted test values under GI50 column indicate activity (see Table 5).

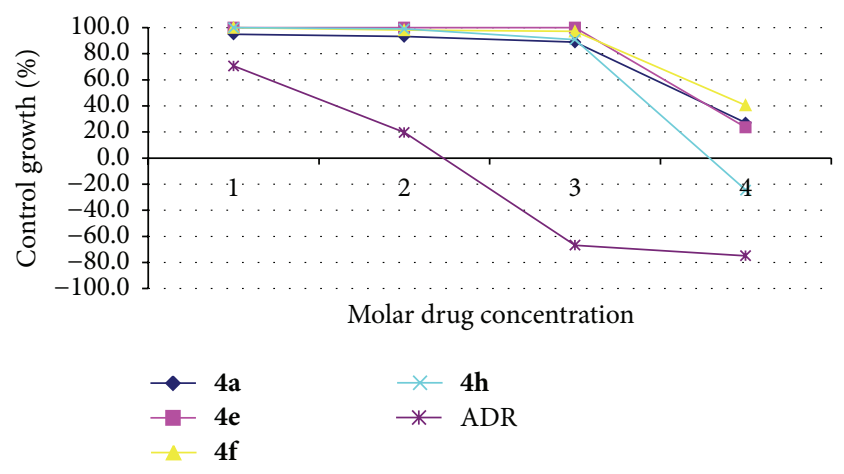

FIGURE 1: In vitro cytotoxic effects of amino acid Schiff bases against human colon cancer cell line Colo205.

\section{Conclusions}

We have developed a convenient, simple, efficient, and ecofriendly green procedure for the synthesis of Schiff bases from $1 \mathrm{H}$-indole-2,3-diones, various amino acids, and thiosemicarbazide under mild reaction conditions in an aqueous medium. Some of the major advantages of this protocol are the ambient conditions, good to excellent yields, simple workup procedure, and use of water as a desirable solvent for chemical reaction for reasons cost, safety, and environmental concerns. Further, use of lemon juice as green catalyst makes this methodology an alternative platform to organic solvent conventional synthesis under the umbrella of environmentally greener and safer processes. The synthesized compounds are found to be active against tested pathogens. The above 
results of antifungal and antibacterial screening establish the fact that thiadiazole substituted indole derivatives can be studied further to explore out newer antimicrobial compounds.

\section{Conflict of Interests}

The authors declare that there is no conflict of interests regarding the publication of this paper.

\section{Acknowledgments}

The authors are thankful to the Dean, FET, MITS, and HOD, Science and Humanities, for providing necessary research facilities in the department. Financial assistance from FET, MITS, is gratefully acknowledged. They are also thankful to the director, The Advanced Centre for Treatment, Research and Education in Cancer (ACTREC), TATA Memorial Centre, Mumbai, India, for anticancer screening, and SAIF, Punjab University, Chandigarh, India, for the spectral analyses of synthesized compounds.

\section{References}

[1] V. K. Redasani, V. S. Kumawat, R. P. Kabra, P. Kansagara, and S. J. Surana, "Applications of green chemistry in organic synthesis," International Journal of ChemTech Research, vol. 2, no. 3, pp. 1856-1859, 2010.

[2] S. Nishimura, A. Takagaki, and K. Ebitani, "Characterization, synthesis and catalysis of hydrotalcite-related materials for highly efficient materials transformations," Green Chemistry, vol. 15, no. 8, pp. 2026-2042, 2013.

[3] M. Poliakoff, J. M. Fitzpatrick, T. R. Farren, and P. T. Anastas, "Green chemistry: science and politics of change," Science, vol. 297, no. 5582, pp. 807-810, 2002.

[4] J. M. DeSimone, "Practical approaches to green solvents," Science, vol. 297, no. 5582, pp. 799-803, 2002.

[5] C. J. Li and T. H. Chan, Organic Reactions in Aqueous Media, Wiley, New York, NY, USA, 1997.

[6] G.-J. Ten Brink, I. W. C. E. Arends, and R. A. Sheldon, "Green, catalytic oxidation of alcohols in water," Science, vol. 287 , no. 5458, pp. 1636-1639, 2000.

[7] U. M. Lindström, "Stereoselective organic reactions in water," Chemical Reviews, vol. 102, no. 8, pp. 2751-2772, 2002.

[8] A. M. Zonouz, I. Eskandari, and H. R. Khavasi, "A green and convenient approach for the synthesis of methyl-6amino-5-cyano-4-aryl-2,4-dihydropyrano[2,3-c] pyrazole-3carboxylates via a one-pot, multi-component reaction in water," Tetrahedron Letters, vol. 53, no. 41, pp. 5519-5522, 2012.

[9] F. Aryanasab, A. Z. Halimehjani, and M. R. Saidi, "Dithiocarbamate as an efficient intermediate for the synthesis of 2-amino1,3,4-thiadiazoles in water," Tetrahedron Letters, vol. 51, no. 5, pp. 790-792, 2010.

[10] F. M. Moghaddam, M. Kiamehr, M. R. Khodabakhshi, Z. Mirjafary, S. Fathi, and H. Saeidian, "A new domino Knoevenagelhetero-Diels-Alder reaction: an efficient catalyst-free synthesis of novel thiochromone-annulated thiopyranocoumarin derivatives in aqueous medium," Tetrahedron, vol. 66, no. 45, pp. 86158622, 2010.
[11] S. S. Pawar, M. S. Shingare, and S. N. Thore, "A novel approach for ligand promoted palladium (II)-catalyzed suzuki coupling of aryl iodides and bromides with arylboronic acid in aqueous media," Letters in Organic Chemistry, vol. 4, no. 7, pp. 486-490, 2007.

[12] Y. Ren and C. Cai, "Iodine catalysis in aqueous medium: an improved reaction system for knoevenagel and nitroaldol condensation," Catalysis Letters, vol. 118, no. 1-2, pp. 134-138, 2007.

[13] V. K. Rao, S. S. Reddy, B. S. Krishna, K. R. M. Naidu, C. N. Raju, and S. K. Ghosh, "Synthesis of Schiff bases in aqueous medium: a green alternative approach with effective mass yield and high reaction rates," Green Chemistry Letters and Reviews, vol. 3, no. 3, pp. 217-223, 2010.

[14] L. Hadjipavlu, J. Dimitra, Geronikaki, and A. Athina, "Thiazolyl and benzo-thiazolyl Schiff base as novel possible lipoxygenase inhibitors and anti-inflammatory agents," Drug Design and Discovery, vol. 15, pp. 199-206, 1998, Chemical Abstracts, vol.129, Article ID 148934, 1998.

[15] B. De and G. V. S. Ramasarma, "Determination of PKa and correlation with the analgesic activity of 5-Oxoimidazolylaminopyrazole-4-carboxaldehydes and their Schiff's bases," Indian Drugs, vol. 36, no. 9, pp. 583-587, 1999.

[16] X.-Y. Luo, J.-Z. Zhao, Y.-J. Lin, and Z.-Q. Liu, "Antioxidative effect of schiff bases with o-hydroxybenzylidene groupon free radical induced hemolysis of human red blood cell," Chemical Research in Chinese Universities, vol. 18, no. 3, pp. 287-289, 2002, Chemical Abstracts, vol. 138, Article ID 247927, 2003.

[17] J. F. M. da Silva, S. J. Garden, and A. C. Pinto, "The chemistry of isatins: a review from 1975 to 1999," Journal of the Brazilian Chemical Society, vol. 12, no. 3, pp. 273-324, 2001.

[18] A. Marin, N. Valls, F. J. Berenguer et al., "Synthesis and anthelmintic activity of carbamates derived from imidazo [2, 1b] $[1,3,4]$ thiadiazole and imidazo [2, 1-b] thiazole," Farmaco, vol. 47, no. 1, pp. 63-75, 1992.

[19] K. C. Joshi, P. Chand, and A. Dandia, "Studies in spiroheterocycles-part II-reactions of fluorine containing indole-2,3-diones with 1,2-phenylendiamines \& 2,3diaminopyridine in different media," Indian Journal of Chemistry B, vol. 23, pp. 743-745, 1984, Chemical Abstracts, vol. 101, 211055, 1984.

[20] E. Palaska, G. Şahin, P. Kelicen, N. T. Durlu, and G. Altinok, "Synthesis and anti-inflammatory activity of 1acylthiosemicarbazides, 1,3,4-oxadiazoles, 1,3,4-thiadiazoles and 1,2,4-triazole-3-thiones," Farmaco, vol. 57, no. 2, pp. 101-107, 2002.

[21] H. Schiff, "Mittheilungenausdem universitatslaboratorium in pisa: eineneue reihe organischer basen," Justus Liebigs Annalen Der Chemie, vol. 131, no. 1, pp. 118-119, 1864.

[22] R. B. Moffett and N. Rabjohn, Organic Synthesis, John Wiley \& Sons, Inc, New York, NY, USA, 1963.

[23] J. H. Billman and K. M. Tai, "Reduction of Schiff bases. II. Benzhydrylamines and structurally related compounds," The Journal of Organic Chemistry, vol. 23, no. 4, pp. 535-539, 1958.

[24] W. A. White and H. Weingarten, "A versatile new enamine synthesis," Journal of Organic Chemistry, vol. 32, no. 1, pp. 213214, 1967.

[25] F. Texier-Boullet, "A simple, convenient, and mild synthesis of imines on alumina surface without solvent," Synthesis, vol. 6-7, pp. 679-681, 1985.

[26] H. Naeimi, F. Salimi, and K. Rabiei, "Mild and convenient one pot synthesis of Schiff bases in the presence of $\mathrm{P}_{2} \mathrm{O}_{5} / \mathrm{Al}_{2} \mathrm{O}_{3}$ as 
new catalyst under solvent-free conditions," Journal of Molecular Catalysis A, vol. 260, no. 1-2, pp. 100-104, 2006.

[27] J. Zhu, L. Chen, H. Wu, and J. Yang, "Highly efficient procedure for the synthesis of Schiff bases using hydrotalcite-like materials as catalyst," Chinese Journal of Chemistry, vol. 27, no. 10, pp. 1868-1870, 2009.

[28] A. Naqvi, M. Shahnawaaz, A. V. Rao, D. S. Seth, and N. K. Sharma, "Synthesis of schiff bases via environmentally benign and energy-efficient greener methodologies," E-Journal of Chemistry, vol. 6, no. 1, pp. S75-S78, 2009.

[29] D. J.-N. Uppiah, M. G. Bhowon, and S. J. Laulloo, "Solventless synthesis of imines derived from diphenyldisulphide diamine or p-Vanillin," E-Journal of Chemistry, vol. 6, no. 1, pp. S195S200, 2009.

[30] M. Gopalakrishnan, P. Sureshkumar, V. Kanagarajan, and J. Thanusu, "New environmentally-friendly solvent-free synthesis of imines using calcium oxide under microwave irradiation," Research on Chemical Intermediates, vol. 33, no. 6, pp. 541-548, 2007.

[31] A. M. Asiri, S. A. Khan, H. M. Marwani, and K. Sharma, "Synthesis, spectroscopic and physicochemical investigations of environmentally benign heterocyclic Schiff base derivatives as antibacterial agents on the bases of in-vitro and density functional theory," Journal of Photochemistry and Photobiology $B$, vol. 120, pp. 82-89, 2013.

[32] S. Patil, S. D. Jadhav, and S. K. Shinde, "CES as an efficient natural catalyst for synthesis of Schiff bases under solvent-free conditions: an innovative green approach," Organic Chemistry International, vol. 2012, Article ID 153159, 5 pages, 2012.

[33] H. Sachdeva, D. Dwivedi, and S. Khaturia, "Aqua mediated facile synthesis of 2-(5/7-fluorinated-2-oxoindolin-3-ylidene)$\mathrm{N}$ - (4-substituted phenyl) hydrazine carbothioamides," Research Journal of Pharmaceutical, Biological and Chemical Sciences, vol. 2, no. 2, pp. 213-219, 2011.

[34] H. Sachdeva and D. Dwivedi, "Lithium-acetate-mediated biginelli one-pot multicomponent synthesis under solvent-free conditions and cytotoxic activity against the human lung cancer cell line A549 and breast cancer cell line MCF7," The Scientific World Journal, vol. 2012, Article ID 109432, 9 pages, 2012.

[35] A. Dandia, R. Singh, H. Sachdeva, and K. Arya, "Microwave assisted one pot synthesis of a series of trifluoromethyl substituted spiro [indole-triazoles]," Journal of Fluorine Chemistry, vol. 111, no. 1, pp. 61-67, 2001.

[36] H. Sachdeva, R. Saroj, S. Khaturia, and D. Dwivedi, "Operationally simple green synthesis of some Schiff bases using grinding chemistry technique and evaluation of antimicrobial activities," Green Processing and Synthesis, vol. 1, no. 5, pp. 469477, 2012.

[37] S. Patil, S. D. Jadhav, and U. P. Patil, "Natural acid catalyzed synthesis of Schiff base under solvent-free condition: as a green approach," Archives of Applied Science Research, vol. 4, no. 2, pp. 1074-1078, 2012.

[38] R. Breslow, "Hydrophobic effects on simple organic reactions in water," Accounts of Chemical Research, vol. 24, no. 6, pp. 159-164, 1991.

[39] S. Otto and J. B. F. N. Engberts, "Hydrophobic interactions and chemical reactivity," Organic and Biomolecular Chemistry, vol. 1, no. 16, pp. 2809-2820, 2003.

[40] J. Chandrasekhar, S. Shariffskul, and W. L. Jorgensen, "QM/MM simulations for Diels-Alder reactions in water: contribution of enhanced hydrogen bonding at the transition state to the solvent effect," The Journal of Physical Chemistry B, vol. 106, no. 33, pp. 8078-8085, 2002.

[41] A. Lubineau and J. Auge, "Water as solvent in organic synthesis," Topics in Current Chemistry, vol. 206, pp. 1-39, 1999.

[42] A. Libineau, J. Auge, and Y. Queneau, "Water-promoted organic reactions," Synthesis, no. 8, pp. 741-760, 1994.

[43] H. Sachdeva, R. Saroj, S. Khaturia, and D. Dwivedi, "Environeconomic synthesis and characterization of some new 1, 2, 4triazole derivatives as organic fluorescent materials and potent fungicidal agents," Organic Chemistry International, vol. 2013, Article ID 659107, 19 pages, 2013.

[44] M. Curini, F. Epifano, F. Maltese, and M. C. Marcotullio, "Novel chiral Schiff base ligands from amino acid amides and salicylaldehyde," Tetrahedron Letters, vol. 43, no. 21, pp. 38213823, 2002.

[45] N. Sari and P. Gürkan, "Some novel amino acid-schiff bases and their complexes synthesis, characterization, solid state conductivity behaviors and potentiometric studies," Zeitschrift fur Naturforschung B, vol. 59, no. 6, pp. 692-698, 2004.

[46] V. Kanagarajan, M. R. Ezhilarasi, and M. Gopalakrishnan, '“One-pot' ultrasound irradiation promoted synthesis and spectral characterization of an array of novel $1,1^{\prime}-\left(5,5^{\prime}-(1,4-\right.$ phenylene) bis(3-aryl-1 $H$-pyrazole-5, $1(4 H, 5 H)$-diyl))diethanones, a bis acetylated pyrazoles derivatives," Spectrochimica Acta A, vol. 78, no. 2, pp. 635-639, 2011.

[47] A. K. Mansour, M. M. Eid, and N. S. A. M. Khalil, "Synthesis and reactions of some new heterocyclic carbohydrazides and related compounds as potential anticancer agents," Molecules, vol. 8, no. 10, pp. 744-755, 2003.

[48] G. Kaur and R. Yadav, "Anti-cancer activities of various heterocyclic containing entites-a review," International Journal of Natural Product Science, vol. 1, article 104, 2012.

[49] I. Ali, A. Haque, K. Saleem, and M. F. Hsieh, "Curcumin-I Knoevenagel's condensates and their Schiff's bases as anticancer agents: synthesis, pharmacological and simulation studies," Bioorganic \& Medicinal Chemistry, vol.21, no. 13, pp. 3808-3820, 2013.

[50] H. Chen and J. Rhodes, "Schiff base forming drugs: mechanisms of immune potentiation and therapeutic potential," Journal of Molecular Medicine, vol. 74, no. 9, pp. 497-504, 1996.

[51] V. Vichai and K. Kirtikara, "Sulforhodamine B colorimetric assay for cytotoxicity screening," Nature Protocols, vol. 1, no. 3, pp. 1112-1116, 2006. 

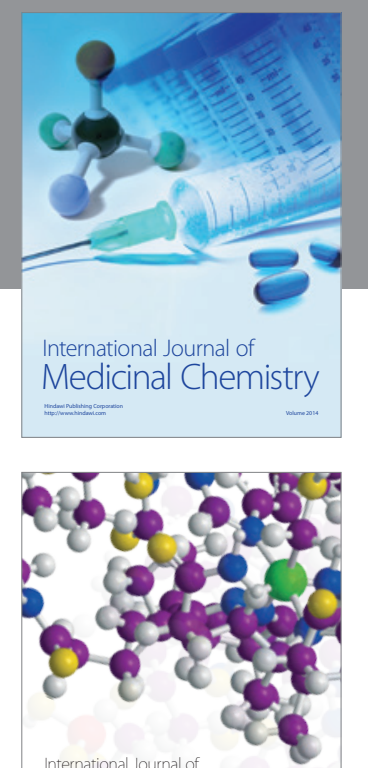

\section{Carbohydrate} Chemistry

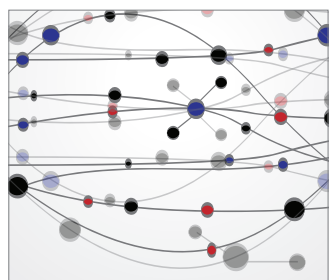

The Scientific World Journal
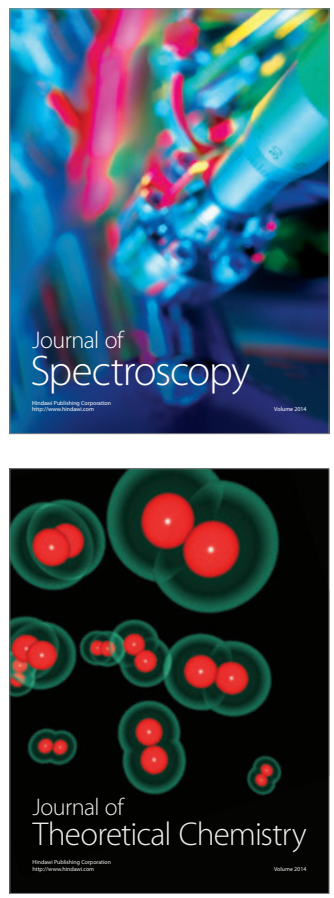
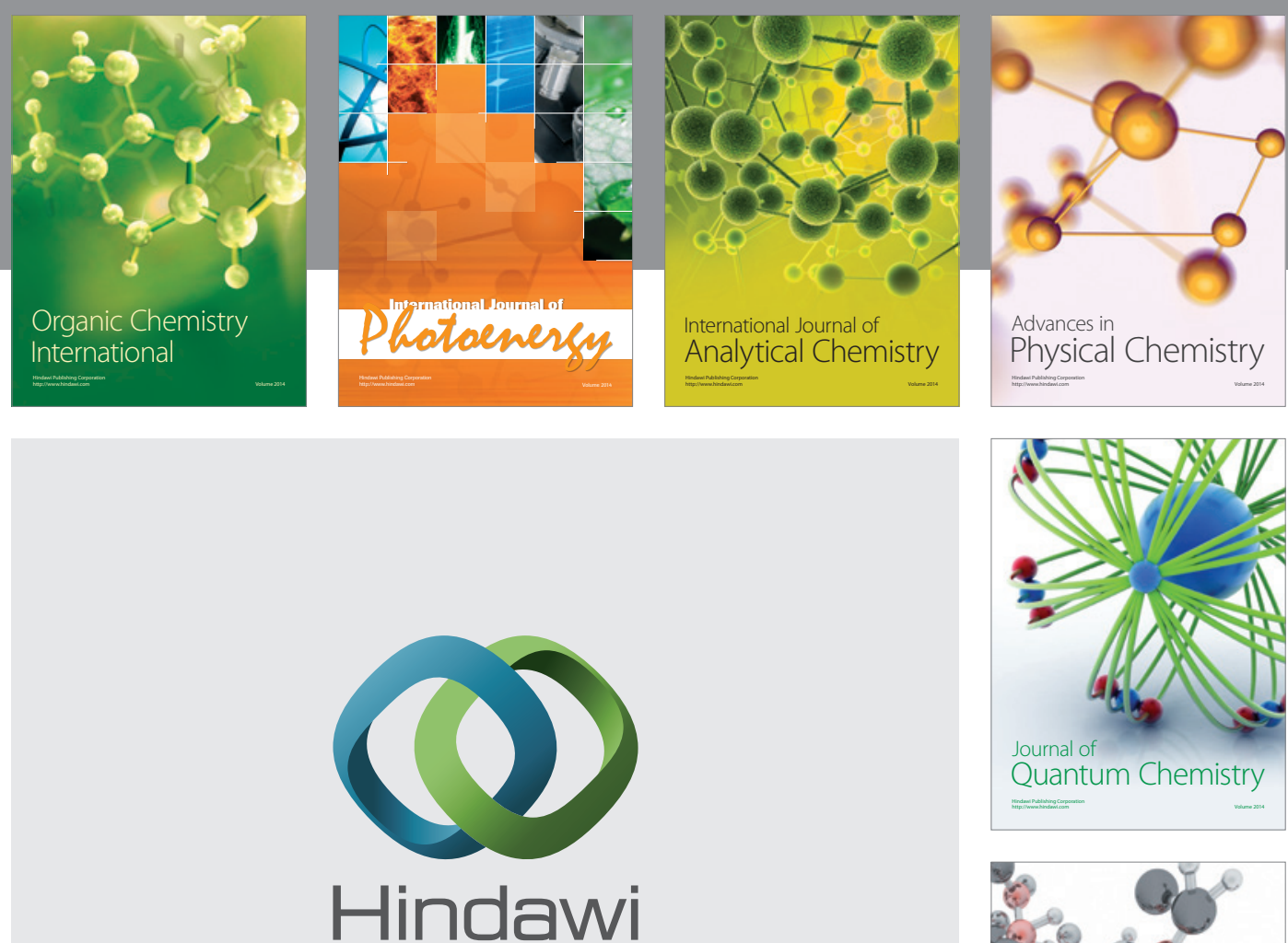

Submit your manuscripts at

http://www.hindawi.com

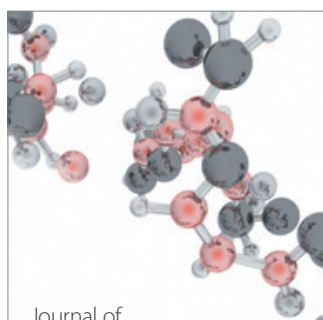

Analytical Methods

in Chemistry

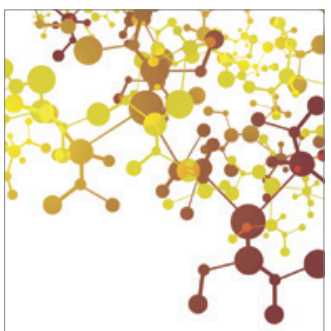

Journal of

Applied Chemistry

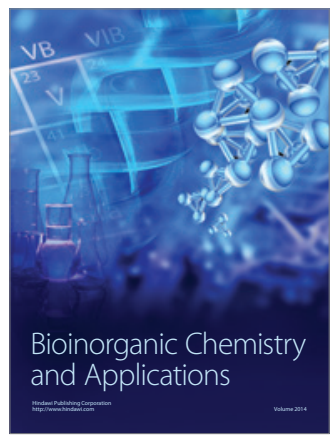

Inorganic Chemistry
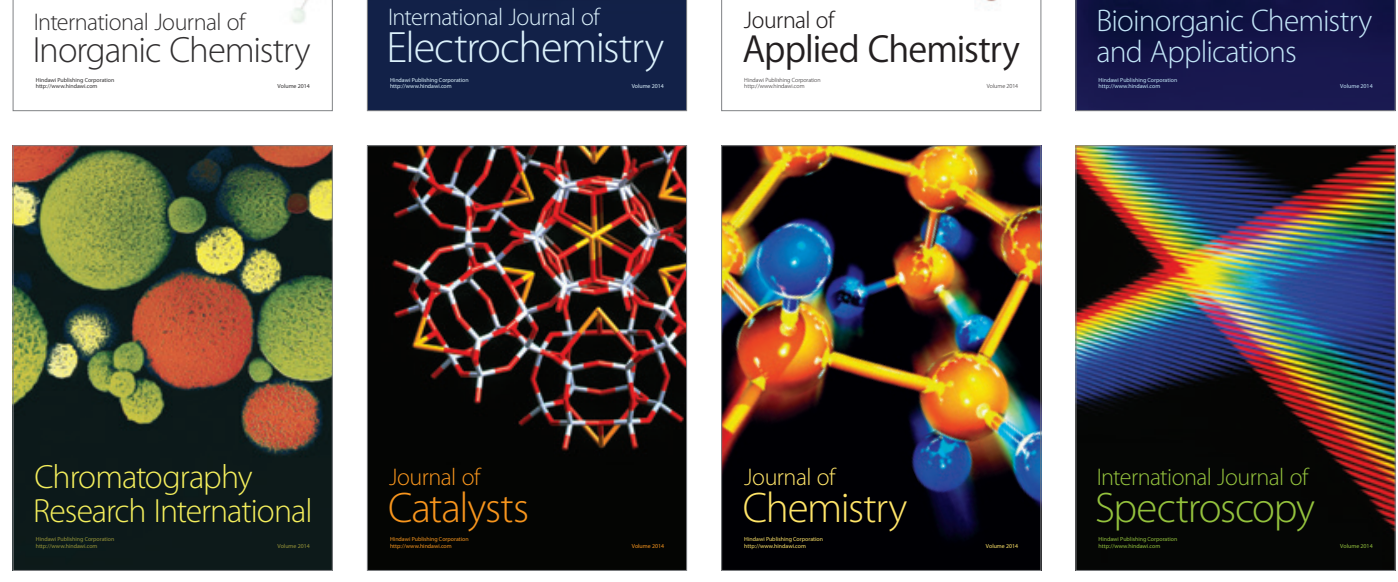